\title{
Papillary glioneuronal tumor: case report and review of literature
}

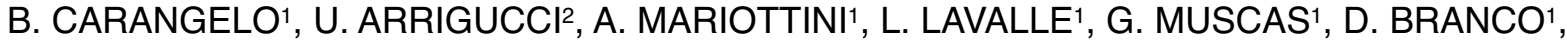 \\ L. LIPPA ${ }^{1}$, G. PERI ${ }^{1}$, M. MUYA $^{1}$, G. COSTANTINO ${ }^{1}$, G. TIEZZI ${ }^{1}$, D. TACCHINI ${ }^{3}$, A. MATURO ${ }^{4}$
}

SUMMARY: Papillary glioneuronal tumor: case report and review of literature.

B. Carangelo, U. Arrigucci, A. Mariottini, L. Lavalle, G. Muscas, D. Branco, L. Lippa, G. Peri, M. Muya, G. Costantino, G. Tiezzi, D. Tacchini, A. Maturo

Papillary glioneuronal tumor (PGNT) is a recently described central nervous system neoplasm that mostly occurs in the supratentorial sy- stem, adjacent to the lateral ventricles.

In 2007, WHO classified PGNT as grade I neuronal-glial tumor because of the characteristic papillary architecture and bipartite (astrocytic and neuronal/neurocytic) cell population. As a newly established entity of mixed glioneuronal tumor family, PGNT attracted extensive attention recently.

In our report we discuss the clinical, neuroradiological and surgical features. The final result is compared with literature data.

KeY Words: Papillary glioneuronal tumor - Mixed glioneuronal tumor - Brain tumor.

\section{Introduction}

Papillary glioneuronal tumor (PGNT) is a recently described central nervous system neoplasm that mostly occurs in the supratentorial system, adjacent to the lateral ventricles. Well-established examples of glioneuronal tumors include DNET (Dysembryoplastic NeuroEpithelial Tumor), ganglioglioma and desmoplastic infantile ganglioglioma. More recently recognized entities include rosette-forming tumor of fourth ventricle, rosette glioneuronal tumor (RGNT)/ glioneuronal tumor with neuropil-like islands and PGNT (1).

In 2007, WHO classified PGNT as grade I neuronal-glial tumor because of the characteristic papillary architecture and bipartite (astrocytic and neuronal/neurocytic) cell population. As a newly established entity of mixed glioneuronal tumor family, PGNT attracted extensive attention recently.

\footnotetext{
Department of Neurosurgery, "Le Scotte" University Hospital of Siena, Siena, Italy

${ }^{2}$ Department of Neuroradiology and Interventional Neurology,

"Le Scotte" University Hospital of Siena, Siena, Italy

${ }^{3}$ Department of Pathology, "Le Scotte" University Hospital of Siena,

Siena, Italy

${ }^{4}$ Department of Surgical Sciences, "Sapienza" University of Rome, Rome, Italy

Corresponding Author: Biagio Carangelo,

e-mail: biagiorobicarangelo@gmail.com

C Copyright 2015, CIC Edizioni Internazionali, Roma
}

It was described in approximately 67 cases to date (Table 1).

Both CT and MRI studies show PGNTs to be circumscribed supratentorial lesions, extending to the cortex and the white matter, or may be more deeply situated, displacing the ventricular system. Cystic alteration are frequent. Solid components are iso/hypointense in $\mathrm{T} 1$ and iso/hyperintense in T2, contrast enhancing in a diffuse, patchy or rimming fashion and may present as mural nodule or plaques within otherwise cystic masses. Calcifications may be seen. Bleeding of lesions are rare but recorded (3), associated with superficial siderosis of neuraxis. Surrounding edema and mass effect are seen in larger lesions. Reported tumor size ranges $1-9 \mathrm{~cm}$ (mean 4,5 cm).

Hystology showed pseudopapillary pattern consisting of a single layer of astrocytic cells surrounding hyalinized blood cells.

Follow-up indicated that this type of tumor had a benign behavior even if recently we found out 5 cases of aggressive PGNT $(1,2,4,5,7,8)$.

\section{Case report}

The patient was a 34 yr old woman with complaints of progressive headache started 2 weeks before and more recently confusion, vomiting and dizziness. She had a va- 


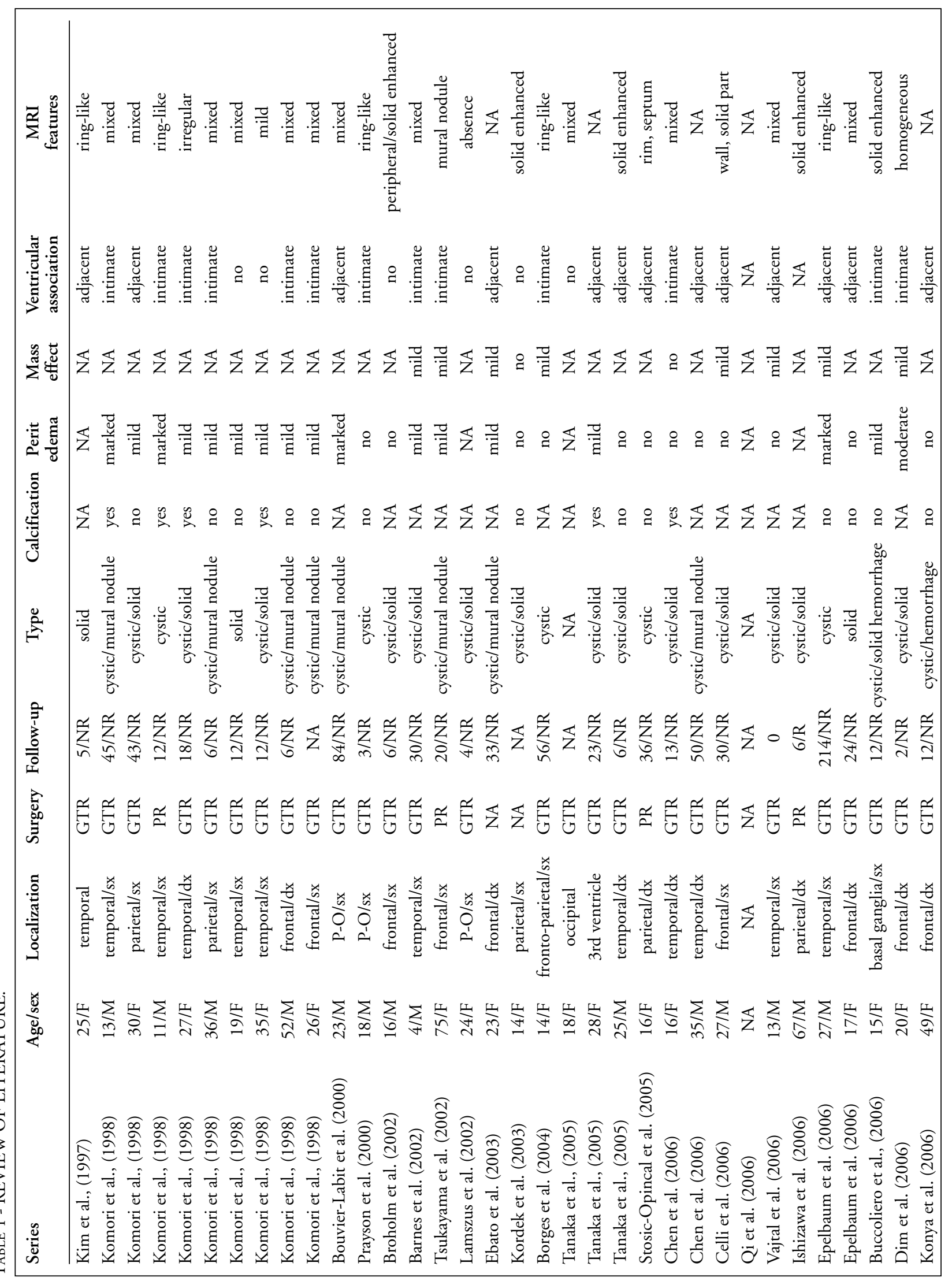


scular operation for aortic coarctation with stenting in 2003. She denied any use of drugs, alcohol and tobacco. Her family history was significant for a pituitary adenoma in her mother. On physical examination, she was found to have deterioration of conscious level, rigor nucalis, right upper arm paresis $(4+/ 5)$, aphasia $(\mathrm{E} 3$, V4, M6). The patient was referred to the hospital for evaluation and imaging studies. The brain CT scan with and without contrast revealed a bulky, iso-iperdense lesion $(50 \times 39 \times 52 \mathrm{~mm})$ in her left basal/polar temporal lobe on post-contrast injection, images showed a round-ring enhancement of the cyst wall with disomogeneous components (Figure 1).

MRI - after administration of contrast enhancement, the cystic portion showed heterogeneous enhancement while the solid component a more homogeneous one. Angio-CT and angio-MRI were also mandatory because of the high suspect of a vascular abnormality that was excluded after the performance of these neuroradiological studies (Figures 2, 3).

The patient underwent a fronto-temporal craniotomy with macroscopically total removal of tumor. Intraope- ratively it appears in most part an hemorrhagic lesion $(90 \%)$ with a minimal solid component $(10 \%)$. All the samples were analyzed and processed. The final histophatological exam that matched with the diagnosis of PGNT documented the presence of "piloid" areas with associated high vascular proliferation and cystic degeneration. It described papillary zones surrounding a vascular core, the vessels showed marked thickening and hyalinization of wall layers, proliferation index (MIB1) $<1 \%$; p53 and EGFR immonoreactivity was not observed (Figures 4, 5). There were no post-operative complications and the patient was discharged after 10 days with complete remission of pre-operative deficits. The postoperative neuroradiological exams showed the complete exeresis of tumor and the absence of further complications. Seizure therapy was prescribed (Levetiraceam). Therefore after 2 months, she was readmitted in our clinic, complaining of headache, pain in the nape of head and lumbar pain. She underwent imaging exams (CT and MRI) that showed new bleeding inside the operative field and an enhanced residual lump (Figure 6). Because of the worsening of neurological status and re-
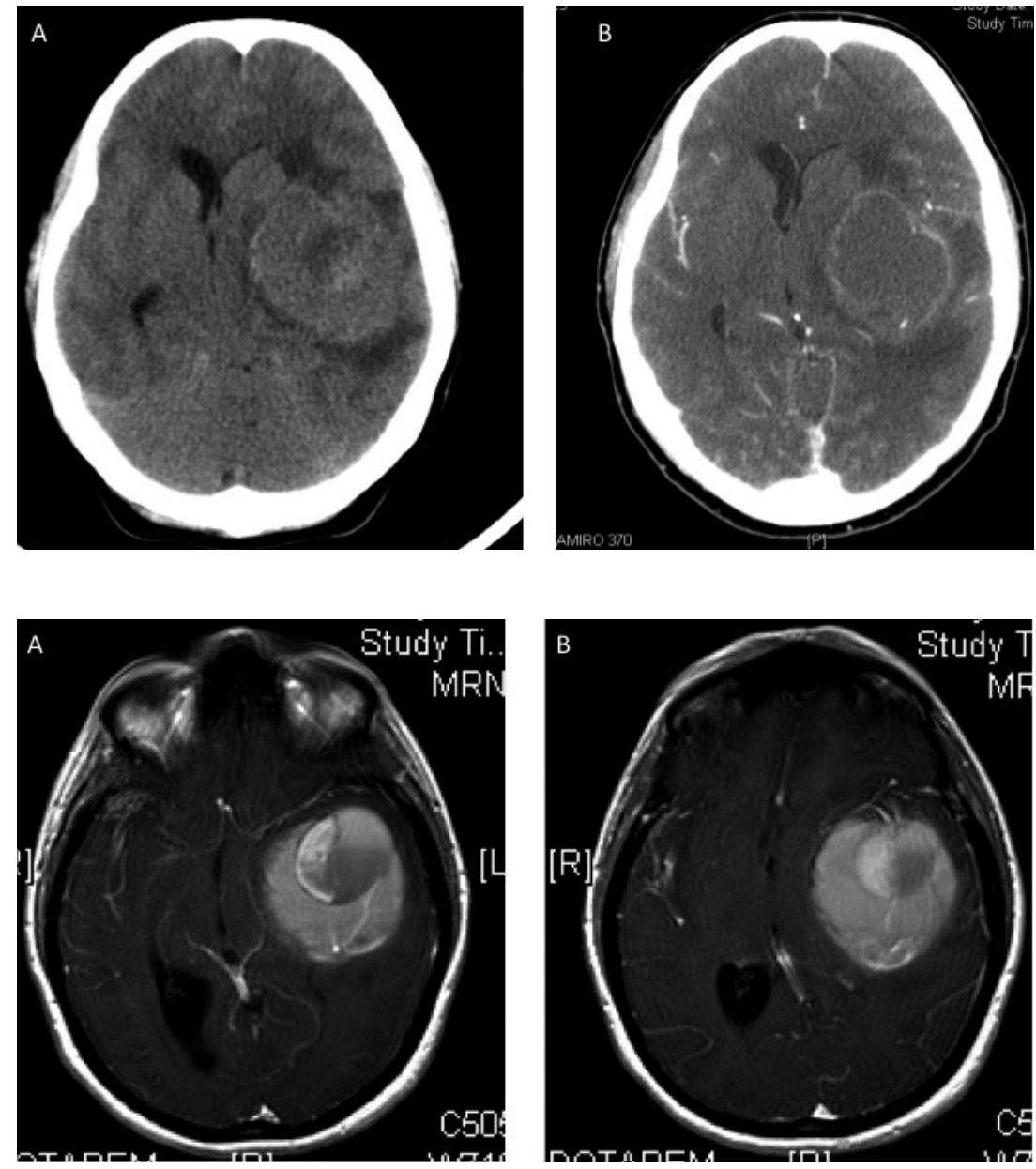

Fig. 2 - A, B) Pre-operative MRI with gadolinum that shows a disomogeneous enhancement of the tumor with solid and cystic components. 

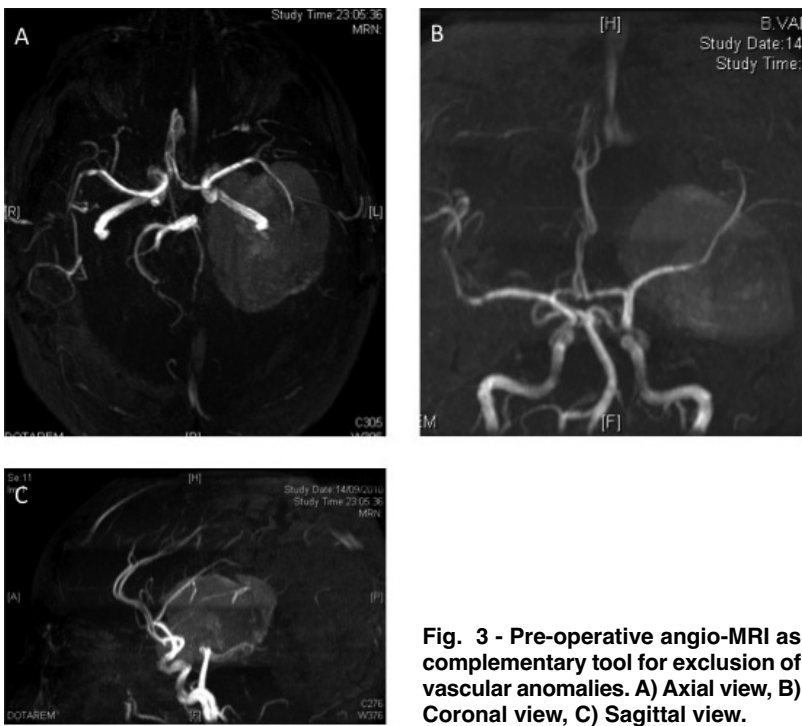

Fig. 3 - Pre-operative angio-MRI as complementary tool for exclusion of vascular anomalies. A) Axial view, B) Coronal view, C) Sagittal view.

currence of tumor, we decided to make a new operation with the evacuation of the blood clot and of lesion. The redo had no complications and reverted the clinical condition to "status quo antea". After the second operation no radio or chemiotherapy were offered to the patient and there were no signs of recurrence in 2 years followup, documented with serial MRIs (Figure 7). Our patient, during last clinic, complained cervical pain with arms radiation that relieved after medical therapies with
NSAIDs and muscle relaxants. After 2 years, the patient came to our emergency department because of generalized seizures. Neurological exam was intact except cervical pain. CT scan and MRI were performed, they showed a minimal presence of bleeding in the operative surgical field, that we decided to treat conservatively. MRI after 3 months showed a good recovery.

However, the last MRI performed (Figure 8) showed a rounded area of hyperintensity within the operative field. This neuroradiological condition was related to a worsening of her epileptic seizures. After performing an fMRI for a further imaging study, we decided to operate the patient making a left temporal lobectomy. The histological exam finally diagnosed a PGNT with a shift from a grade I to grade II. The operation didn't have complications and the patient did well as it was confirmed by the radiological control (Figure 9). We chose not to subject the patient to further therapies, rather to intensify strictly the clinical and neuroimaging controls. This histological finding raises questions about the benign nature of the lesion. We also noticed that the literature reports just describe the aggressiveness of this tumour from the beginning of its presentation.

\section{Discussion}

Papillary glioneuronal tumor is a distinct entity recently included in the Neuronal and Mixed glial
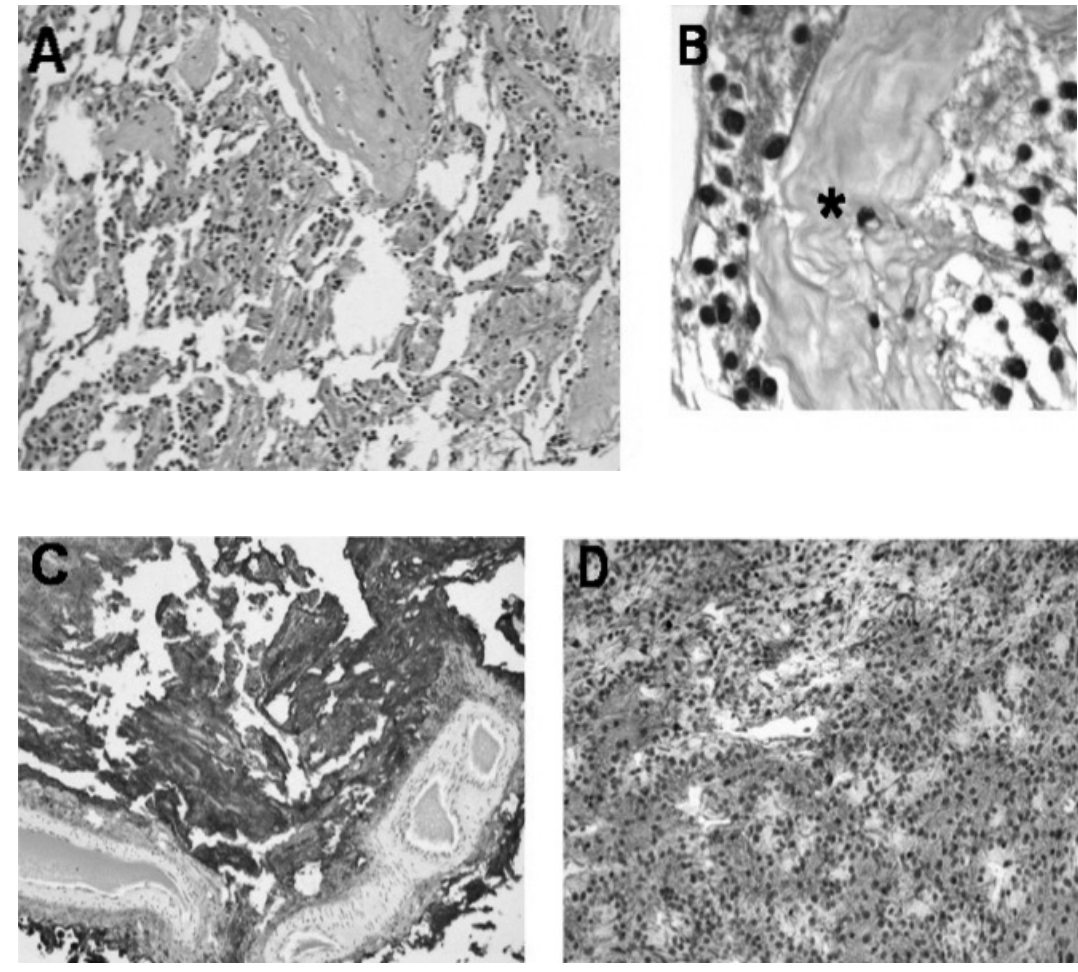

Fig. 4 - Histopathological exam. A) Pseudopapillary pattern of the neoplasm. B) Detail of a "papilla", showing a hyanalized core (asterisk), linked with oligodendroglial-like cells. A, B) Hematoxylin and eosin, original magnification: A, x 100; B, x 100 .

Fig. 5 - Histopathological exam. C) Tumor cells immediately surrounding vessels are GFAP-positive. D) Synaptophysin-positive neurocytes, stained brown by diaminobenzydine, are intermingled with glial negative cells. C, D) Immunohistochemistry, Avidin-Biotin method, original magnification: C, x 50; D, $x 100$. 

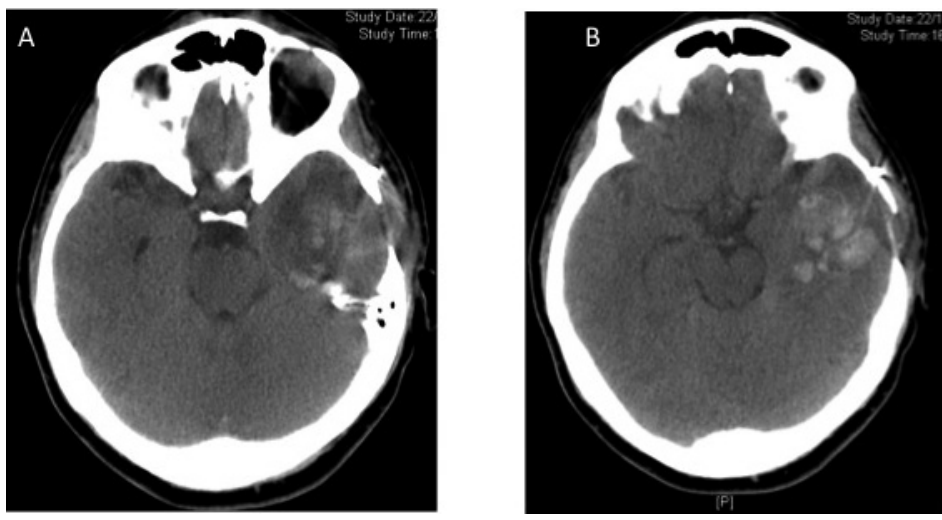

Fig. 6 - A, B) 2-month CT scan showing the new bleeding in the left temporal lobe.
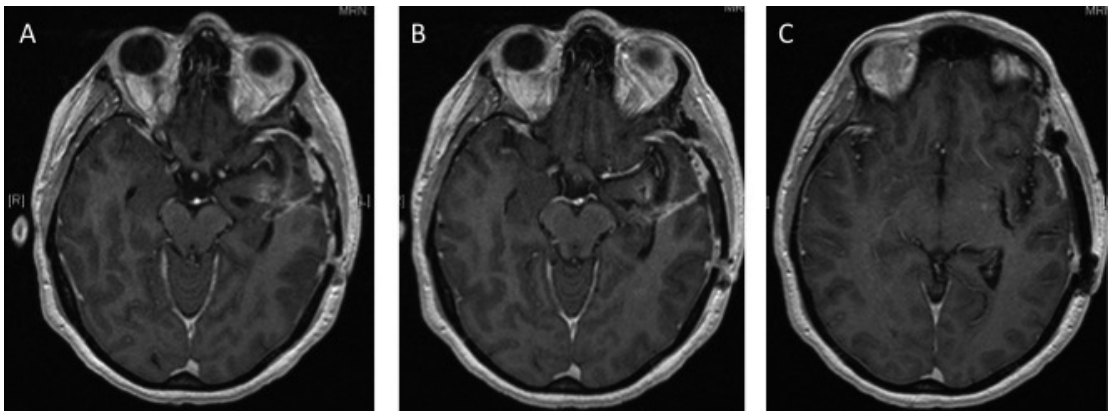

Fig. 7 - A, B, C) 1-month MRI after the second operation that shows neither residual not recurrence of the tumor.
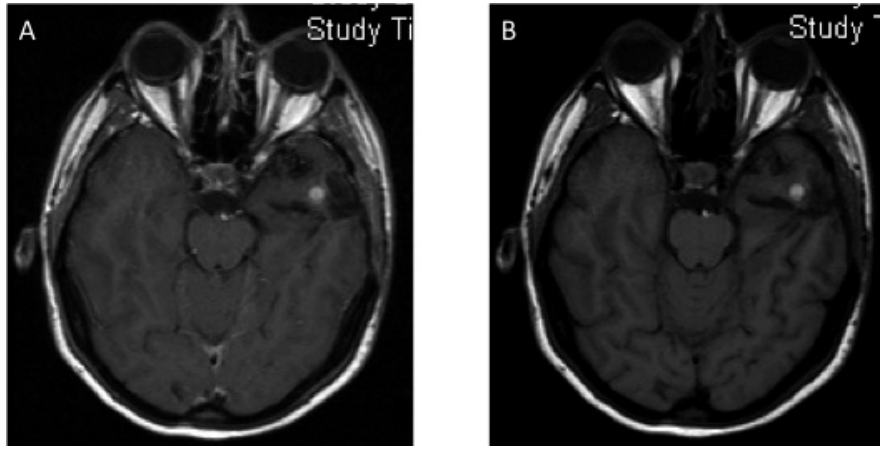

Fig. 8 - A, B) 2-year MRI. Little hyperintensity in T1 sequence, in the temporal left region.
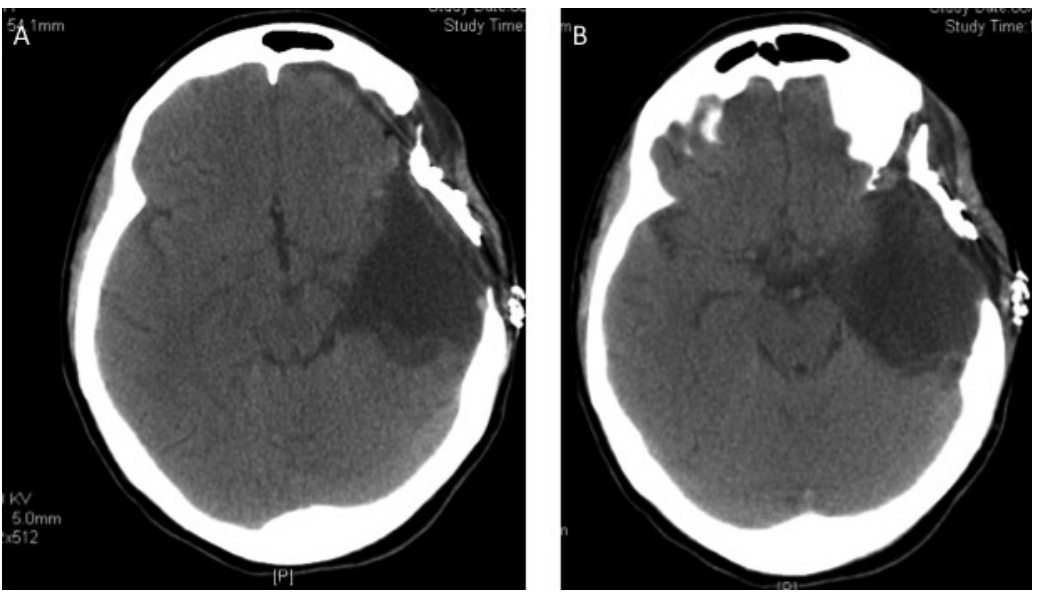

Fig. 9 - A, B) Control CT scan after the third operation. 
group of CNS tumors in the $2007 \mathrm{WHO}$ classification. These are slow-growing indolent tumors and involve patients over a wide range of age varying from 4 to 75 years. Komori et al., as first, reported in 1998 this entity as PGNT (papillary glioneuronal tumor). A similar hystologic case was reported by Kim et al. in 1997 as pseudopapillary neurocytoma. The glial component is astrocytic, characterized by pseudopapillary formation and featuring conspicuous hyalinized mixed vasculature. The vessels are enclosed by a uniform, single, or pseudostratified layer of small cuboidal cells with round reticular nuclei without atypia and scant cytoplasm. These cells are uniformly immunoreactive for GFAP and S-100 antibodies and in some cases they show immunostaining with synaptophysin antibodies. The interpapillary spaces contain small round neuronal cells with perinuclear halos, resembling oligodendrocytes that have been shown to stain for anti-oli and synaptophysin antibodies. Other pertinent findings, seen in most cases, included the presence of Rosenthal fibers. Proliferative indices, as determined by immunostaining with Ki-67, have uniformly been shown to be low. The patients are asyntomatic or complain of mild neurologic symptoms. With the addition of our case the total number of published cases concerning PGNTs exceeds 68. Most of the currently recognized glioneuronal tumors has a benign course. There are, however, examples of more aggressive glioneuronal tumor described in the literature. Adam C et al., described the first case of aggressive variant of papillary glioneuronal tumor, doubting about the complete benignity of this variant. Then also Newton HB and Javahery RJ described a case of aggressive PGNTs, while Myung JK had a death because of the disease progression. In our case we have not an aggressive component but a recurrence of PGNT.

The hystogenesis of papillary glioneuronal tumor is uncertain and its origin from multipotent precursor cells capable of divergent glioneuronal differentiation has been suggested by its common periventricular location, probably arising from subependymal stem cells. The malignant or anaplastic component of glioneuronal tumor may be the glial portion, neuronal portion, or both $(6$, 9). The more superficially located ones probably originate from the secondary germinal layer. Expression of PDGF R alfa olig 2 and Nestin by the tumor cells indicates an origin from subependimal progenitor cells. Vaquero et al in a single genetic study published reveal games structural alteration in chromosoma 7 with break points at $7 \mathrm{p} 22$ and amplification at 7p14-q12 (10). There are no characteristic imagings of the tumor that could be specifically indicative of an aggressive nature in our case. In effect PGNTs, like other neurological tumors do not always have a strictly benign course.

\section{References}

1. Adam C, Polivka M, Carpentier A, Carpentier A, George B, Gray F. Papillary glioneuronal tumor: not always a benign tumor? Clin Neuropathol. 2007;26:119-124.

2. Agarwal S, Sharma MC, Singh G, Suri V, Sarkar C, Garg A, Kumar R, Chandra PS. Papillary glioneuronal tumor- a rare entity: report of four cases and brief review of literature. Childs Nerv Syst. 2012;28(11):1897-904.

3. Buccoliero AM, Giordano F, Mussa F, Taddei A, Genitori L, Taddei GL. Papillary Glioneuronal tumor radiologically mimicking a cavernous emangioma with hemorragic onset. Neurophatology. 2006;26:206-211.

4. Javahery RJ, Davidson L, Fangusaro J, Finlay JL, Gonzalez-Gomez I, McComb JG. Aggressive variant of papillary glioneuronal tumor. Report of 2 cases. J Neurosurg Pediatr. 2009;3:4652.

5. Kim DH, Suh YL. Pseudopapillary neurocytoma of temporal lobe with glial differentiation. Acta Neuropathol. 1997;94:187191.
6. Komori T, Scheithauer BW, Anthony DC, Rosemblum MK, McLendon RE, Scott RM, Okazaki H, Kobayashi M. Papillary glioneuronal tumor: a new variant of mixed neuronalglial neoplasm. Am J Surg Pathol. 1998;22:1171-1183.

7. Myung JK, Byeon SJ, Kim B, Suh J, Kim SK, Park CK, Chung CK, Chang KH, Park SH. Papillary glioneuronal tumors: a review of clinicopathologic and molecular genetic studies. Am J Surg Pathol. 2011;35:1794-1805.

8. Newton HB, Dalton J, Ray Chaudhury A, Gahbauer R, McGregor J. Aggressive papillary glioneuronal tumor: case report and literature review. Clin Neuropathol. 2008;27:317-324.

9. Tanaka Y, Yokoo H, Komori T, Makita Y, Ishizava T, Hirose T, Ebato M, Shibara J, Tsukayama C, Shibuya M, NAzakato Y. A distinct pattern of Oligo2-positive cellular distribution in papillary glioneuronal tumors: a manifestation of the oligodendroglial phenotype? Acta Neuropathol. 2005;110:39-47.

10. Vaquero J, Coca S. Atypical papillary glioneuronal tumor. J Neurooncol. 2007;83:319-323. 